\title{
Rifampicin-Resistant Disseminated Tuberculosis in an Immunocompetent Adolescent Male Presenting with Retropharyngeal Abscess and Spinal Involvement
}

\author{
Arghya Das ${ }^{1}$ Vineeta Gupta² Shampa Anupurba \\ 1Department of Microbiology, Institute of Medical Sciences, \\ Banaras Hindu University, Varanasi, Uttar Pradesh, India \\ ${ }^{2}$ Department of Pediatrics, Institute of Medical Sciences, Banaras \\ Hindu University, Varanasi, Uttar Pradesh, India
}

\author{
Address for correspondence Shampa Anupurba, MD, Department \\ of Microbiology, Institute of Medical Sciences, Banaras Hindu \\ University, Varanasi, Uttar Pradesh, 221005, India \\ (e-mail: shampa_anupurba@yahoo.co.in).
}

J Lab Physicians 2021;13:277-279.

\author{
Abstract \\ Keywords \\ - Spinal TB \\ - retropharyngeal \\ abscess \\ - Xpert MTB/RIF \\ - rifampicin resistance
}

Retropharyngeal abscess is a rare manifestation in spinal tuberculosis. Early clinical diagnosis followed by microbiological confirmation and effective treatment is crucial to avoid irreversible damage to the spine. Here, we report a case of disseminated tuberculosis in an immunocompetent adolescent male who presented with retropharyngeal abscess, multifocal involvement of the spine, and skin tuberculids. Xpert MTB/RIF assay in this patient facilitated early lifesaving treatment by detecting rifampicin-resistant Mycobacterium tuberculosis (MTB) in the clinical specimen.

\section{Introduction}

Mycobacterium tuberculosis (MTB) can affect any part of the body, including bones. Skeletal tuberculosis (TB) constitutes less than $2 \%$ of all TB cases. ${ }^{1}$ The spine being the most common site of osseous involvement, accounts for approximately $50 \%$ of skeletal TB cases. ${ }^{2}$ Tubercular spondylitis of cervical spine may rarely present with retropharyngeal abscess. ${ }^{3}$

In patients with suspected TB, the use of NAATs, specifically Xpert MTB/rifampicin (RIF) cartridge-based nucleic acid amplification testing (CBNAAT), has revolutionized the diagnosis with its ability to detect MTB while simultaneously assessing rifampicin resistance within 2 hours. Here, we report the case of an immunocompetent adolescent male who was found to have multifocal involvement of spine and retropharyngeal abscess, and emphasize the importance of Xpert MTB/RIF for early detection of rifampicin resistance.

\section{Case Report}

A previously healthy 12-year-old Indian male was admitted in our hospital with disabling back pain, numbness in

published online July 10, 2021
DOI https://doi.org/

$10.1055 / \mathrm{s}-0041-1730754$ ISSN 0974-2727 bilateral lower limbs for 6 months, and difficulty in swallowing for 3 months. History of low grade, intermittent fever for 6 months with significant weight loss were also present. There was no associated history of cough, chest pain, hemoptysis, dyspnea, or stridor. Bladder and bowel habits were normal. The patient had recent exposure to two pulmonary tuberculosis cases in his neighborhood.

On general examination, the boy was febrile, emaciated with severe pallor, and bilateral enlargement of cervical lymph nodes which were mobile, discrete, and nontender. Axillary and inguinal lymph nodes were also enlarged. There were gibbus on the midspine and multiple papulonecrotic swellings over hands and legs (-Fig. 1). Examination of the nervous system revealed decreased power in bilateral lower limbs with muscle wasting, hypotonia, reduced deep tendon reflexes, and equivocal plantar response. There was no sensory impairment. Examination of other systems was unremarkable. However, oropharyngeal examination revealed a small fluctuant swelling located centrally on the posterior pharyngeal wall.

Chest X-ray showed no obvious abnormality except few patchy changes. Ultrasonography of abdomen revealed

(C) 2021. The Indian Association of Laboratory Physicians

This is an open access article published by Thieme under the terms of the Creative Commons Attribution-NonDerivative-NonCommercial-License, permitting copying and reproduction so long as the original work is given appropriate credit. Contents may not be used for commercial purposes, or adapted, remixed, transformed or built upon. (https://creativecommons.org/licenses/by-nc-nd/4.0/). Thieme Medical and Scientific Publishers Pvt. Ltd. A-12, 2nd Floor, Sector 2, Noida-201301 UP, India 

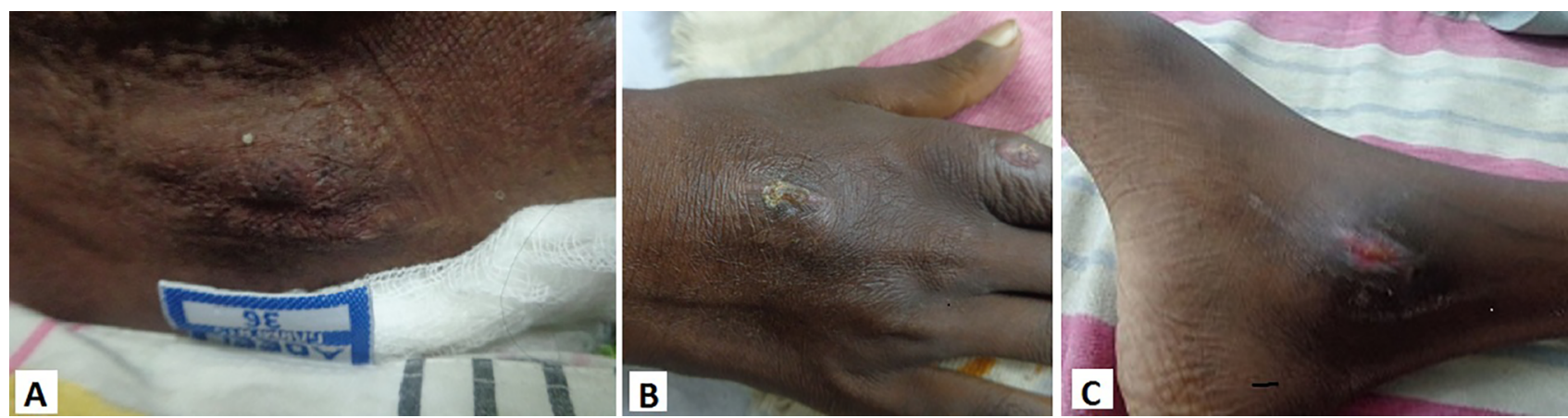

Fig. 1 (A) Gibbus deformity on the spine, (B) papulonecrotic swelling on the dorsum of right hand, (C) papulonecrotic swelling on the medial aspect of right ankle.

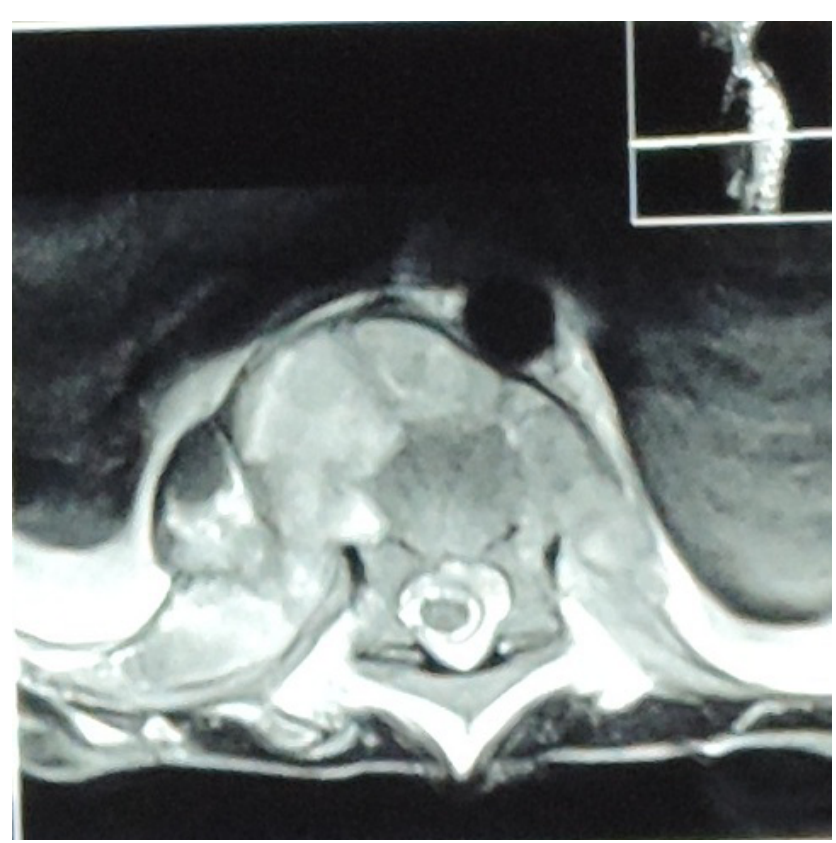

Fig. 2 MRI showing abscess in the body of vertebrae.

thickened mesentery with multiple enlarged mesenteric lymph nodes. Also, MRI of spine revealed caries spine involving D8-D9 vertebra with multiple abscesses along D-4, D-7, and D-9 (-Fig. 2). The retropharyngeal abscess was drained under local anesthesia and pus was sent for microbiological examination. Xpert MTB/RIF assay (CBNAAT) on pus detected presence of MTB with rifampicin resistance. MTB was isolated from the same specimen on Lowenstein-Jensen (LJ) medium after 5th week of culture, but smear microscopy following ZN staining was negative. The second line-line probe assay (SL-LPA) conducted on the culture isolate detected no additional resistance to fluoroquinolones or second-line injectable drugs. Blood culture report was sterile after 7 days of incubation. The patient was HIV negative and microbiological examinations on induced sputum were negative for MTB.

The patient underwent 6 months of intensive phase treatment with levofloxacin, kanamycin, ethionamide, cycloserine, pyrazinamide, and ethambutol, followed by 18 months of continued phase treatment with levofloxacin, ethionamide, cycloserine, and ethambutol, and was declared clinically recovered after periodic follow-up visits. All the constitutional symptoms disappeared and skin tuberculids were healed. However, the spinal deformity called for orthopedic intervention.

\section{Discussion}

Although TB is primarily a disease of the lungs, extrapulmonary TB is an equally important entity in modern clinical practice. Clinical expertise is of paramount importance for making provisional diagnosis of extrapulmonary $\mathrm{TB}$ as well as choosing adequate diagnostic tests. Because of the paucibacillary nature of the lesions, microbiological diagnosis is often delayed in such cases. Despite the absence of any obvious abnormality in chest X-ray in our case, a careful assessment of history, including the history of exposure and general examination revealing lesions on the skin raised the suspicion of TB, which was more evidenced after the MRI report strongly suggested spinal TB.

In spinal tuberculosis, infection sets in the subchondral region of vertebral bodies, progressing to result in the collapse of vertebral bodies, which may be manifested as wedging, kyphosis, sharp angulation, or gibbus deformity. ${ }^{4}$ An abscess is usually formed inside the vertebral bodies with collection of exudates. When the vertebral bodies cannot support the weight transmitted, they collapse and squeeze out the internal contents of the abscess, which then track into the surrounding tissues to form paravertebral abscess. ${ }^{.}$The exudative material from the cold abscess of the cervical spine may sometimes traverse into the retropharyngeal space through the ruptured anterior and posterior longitudinal ligaments, thus forming retropharyngeal abscess, which is a very rare presentation. ${ }^{4,6}$

Early stages of spinal TB may present with constitutional symptoms such as low-grade fever, weight loss, and back pain. In addition, dysphagia and torticollis may be manifested in patients with retropharyngeal abscess. Our patient presented with all these symptoms except torticollis. Paraplegia or paraparesis is often the first clinical manifestation of the spinal TB. The neurological deficit in such cases is the result of external compression of the spinal cord by extradural abscess, subluxation or collapse of vertebral bodies, granuloma, and stretching of the spinal cord over bony ridges. ${ }^{7}$ Early neurological deficit was also evident in our patient in terms of bilateral progressive numbness in lower limbs. 
The gold standard for diagnosis of any form of TB is culture of MTB which was positive in our case, but it took time. On other hand, application of Xpert MTB/RIF improved the diagnostic accuracy in terms of rapidity, exclusion of nontuberculous mycobacteria, and detection of rifampicin resistance. While ultrasonography depicted intra-abdominal involvement, MRI was helpful in differentiating between abscess and granulation tissue and localizing the multiple lesions along the spine.

This case illustrates the importance of high index of suspicion of tuberculosis when evaluating a patient with back pain and dysphagia even with no pulmonary symptom or sign. Early imaging and appropriate microbiological testing using Xpert MTB/RIF (especially for early detection of rifampicin resistance) can avoid diagnostic delay and facilitate early effective treatment.

\section{Conclusion}

A high index of suspicion should be kept for tuberculosis in cases of retropharyngeal abscess associated with spinal involvement to avoid diagnostic delays, which may lead to irreversible damage. The microbiological workup using Xpert $\mathrm{MTB} / \mathrm{RIF}$, as a rapid tool for detection of MTB as well as rifampicin resistance, showed the utility of the test for analyzing nonrespiratory specimen and also facilitated early and lifesaving treatment in this case.

\section{Authors' Contributions}

A.D. contributed to developing the concept and design of the report, definition of intellectual content, literature research, and manuscript preparation and editing. V.G. contributed in the definition of intellectual content and manuscript editing and review. S.A contributed in manuscript editing and review, and is also the guarantor for the report.

\section{Conflicts of Interest}

None declared.

\section{References}

1 de Araujo PS, de Melo HR, de Melo FL, et al. Multifocal skeletal tuberculosis in an immunocompetent patient: a case report. BMC Infect Dis 2015;15:235

2 Garg RK, Somvanshi DS. Spinal tuberculosis: a review. J Spinal Cord Med 2011;34(5):440-454

3 Patil C, Patil RK, Deshmukh P, Singhal S, D'Souza B. Tuberculous retropharyngeal abscess without cervical spine TB. Asian Pac J Trop Med 2011;4(3):251-252

4 Garg A, Wadhera R, Gulati SP, Kishore D, Singh J. Giant retropharyngeal abscess secondary to tubercular spondylitis. Indian J Tuberc 2009;56(4):225-228

5 Bhargava SK, Gupta S. Large retropharyngeal cold abscess in an adult with respiratory distress. J Laryngol Otol 1990;104(2):157-158

6 Ekka M, Sinha S. Retropharyngeal abscess as a rare presentation of pulmonary tuberculosis. Lung India 2015;32(3):262-264

7 Gorse GJ, Pais MJ, Kusske JA, Cesario TC. Tuberculous spondylitis. A report of six cases and a review of the literature. Medicine (Baltimore) 1983;62(3):178-193 